\title{
Erratum
}

\section{Erratum to: Cell-Matrix De-Adhesion Dynamics Reflect Contractile Mechanics}

\author{
Shamik Sen and Sanjay Kumar \\ Department of Bioengineering, University of California, Berkeley, Berkeley, CA 94720-1762, USA
}

\section{Erratum to: Cellular and Molecular Bioengineering DOI 10.1007/s12195-009-0057-7}

The equation in Figure 1d is displayed incorrectly and should read as follows (note the argument of the exponential):

$$
A_{\text {normalized }}=1-\frac{1}{1+e^{\left(t-\tau_{1}\right) / \tau_{2}}}
$$

Address correspondence to Sanjay Kumar, Department of Bioengineering, University of California, Berkeley, Berkeley, CA 947201762, USA. Electronic mail: skumar@berkeley.edu

The online version of the original article can be found under doi: 10.1007/s12195-009-0057-7.

1865-5025/11/0003-0122/0 (C) 2011 Biomedical Engineering Society 